\title{
The Fine-Tuning Argument and the Simulation Hypothesis
}

Forthcoming in Think

Moti Mizrahi

St. John's University

Abstract. In this paper, I propose that, in addition to the multiverse hypothesis, which is commonly taken to be an alternative explanation for fine-tuning, other than the design hypothesis, the simulation hypothesis is another explanation for fine-tuning. I then argue that the simulation hypothesis undercuts the alleged evidential connection between 'designer' and 'supernatural designer of immense power and knowledge' in much the same way that the multiverse hypothesis undercuts the alleged evidential connection between 'fine-tuning' and 'fine-tuner' (or 'designer'). If this is correct, then the fine-tuning argument is a weak argument for the existence of God.

Keywords: design hypothesis; fine-tuning argument; multiverse hypothesis; simulation hypothesis

\section{Introduction}

The Fine-Tuning Argument (henceforth, FTA) is based on the observation that the universe is 'fine-tuned' for life. To say that the universe is fine-tuned for life is to say that the values of 
certain cosmic parameters are such that, were they slightly different, life would not have existed in the universe. Those cosmic parameters are the following ${ }^{1}$ :

$\begin{array}{ll}\text { Parameter } & \text { Actual value } \\ \mathrm{M}_{\mathrm{p}} \text { (mass of the proton) } & 938.28 \mathrm{MeV} \\ \mathrm{M}_{\mathrm{n}} \text { (mass of the neutron) } & 939.57 \mathrm{MeV} \\ \mathrm{c} \text { (the speed of light) } & 2.99792458 \times 10^{8} \mathrm{~m}^{1} \mathrm{~s}^{-1} \\ \mathrm{G} \text { (the Newtonian gravitational constant) } & 6.6742 \times 10^{-11} \mathrm{~m}^{3} \mathrm{~kg}^{-1} \mathrm{~s}^{-2}\end{array}$

'Given this extremely improbable fine-tuning', the argument goes, 'we should think it much more likely that God exists than we did before we learned about fine-tuning, ${ }^{2}$ More formally, the FTA can be stated as follows:

(1) $\mathrm{P}(\mathrm{E} \mid \mathrm{K} \& \sim \mathrm{D}) \approx 0$

(2) $\mathrm{P}(\mathrm{E} \mid \mathrm{K} \& \mathrm{D})>>0$

(3) $\mathrm{P}(\mathrm{D} \mid \mathrm{K}) \gg>\mathrm{P}(\mathrm{E} \mid \mathrm{K} \& \sim \mathrm{D})$

(4) $\therefore \mathrm{P}(\mathrm{D} \mid \mathrm{E} \& \mathrm{~K})>>0$

$\mathrm{K}=$ Many of the initial conditions and free parameters of a universe need to be finely tuned in order for the development of life in that universe to be possible. $\mathrm{E}=$ The universe is indeed fine-tuned for life. 
$\mathrm{D}=$ A supernatural designer of immense power and knowledge exists. ${ }^{3}$

The following are two familiar objections to the FTA:

Objection 1. The multiverse hypothesis. According to the multiverse hypothesis, there are many (perhaps infinitely many) universes. ${ }^{4}$ This hypothesis is an alternative explanation for fine-tuning because, if it is true, then the probability of a fine-tuned universe is high, not low, and thus there is no need to invoke a supernatural entity in order to explain finetuning. 5

Objection 2. Fine-tuning is not improbable. According to this objection, the FTA fails as a Bayesian argument from small probabilities because 'there is no natural way to quantify the intrinsic improbability of the known "coincidences"., 6

In response to Objection 1, some have argued that the multiverse hypothesis may explain why some universe is fine-tuned for life but not why this universe is fine-tuned for life. ${ }^{7}$ To this response to Objection 1, Manson and Thrush have replied by arguing that the 'this universe' objection to the multiverse hypothesis fails for the same reason that the 'this planet' objection fails as an objection to any explanation of the origin of life on Earth. ${ }^{8}$

More recently, Weisberg has raised another objection against the FTA. For Weisberg, the FTA can be stated as follows 9 :

(1) $\mathrm{P}(\mathrm{E} \mid \mathrm{D})>\mathrm{P}(\mathrm{E} \mid \sim \mathrm{D})$

(2) If $\mathrm{P}(\mathrm{E} \mid \mathrm{H})>\mathrm{P}(\mathrm{E} \mid \sim \mathrm{H})$ then E supports $\mathrm{H}$ over $\sim \mathrm{H}$ [The Likelihood Principle] 


\section{(3) $\therefore$ E supports D over $\sim \mathrm{D}$}

Weisberg's objection is that we have known E (i.e., that the universe allows for the existence of life) for some time. For this reason, D does not predict $\mathrm{K}$ any more strongly than $\sim \mathrm{D}$ does. Given the old piece of evidence $\mathrm{E}$ and the new piece of evidence $\mathrm{K}$, Weisberg argues, the FTA must be revised as follows:

(1) $\mathrm{P}(\mathrm{K} \mid \mathrm{D} \& \mathrm{E})>\mathrm{P}(\mathrm{K} \mid \sim \mathrm{D} \& \mathrm{E})$

(2) If $\mathrm{P}(\mathrm{E} \mid \mathrm{H})>\mathrm{P}(\mathrm{E} \mid \sim \mathrm{H})$ then $\mathrm{E}$ supports $\mathrm{H}$ over $\sim \mathrm{H}$ [The Likelihood Principle]

(3) $\therefore$ K supports D over $\sim \mathrm{D}$ (given E)

But, in that case, Weisberg argues, the first premise is false. Given the old piece of evidence that our universe does allow for the existence of life, fine-tuning is equally likely whether there is a supernatural designer or not. White ${ }^{10}$ replies to Weisberg's objection and a lively debate ensued. $^{11}$

In this paper, however, I argue that there is another, heretofore unappreciated, problem with the FTA. The aforementioned objections target the alleged evidential connection between 'fine-tuning' and 'fine-tuner' (or 'designer'). For example, according to Objection 1, if the multiverse hypothesis is true, then fine-tuning is not strong evidence for the existence of a supernatural designer, since fine-tuning can be explained without postulating a supernatural designer of immense power and knowledge. To put it another way, since the FTA is supposed to be a Bayesian argument, and hence an instance of defeasible inference, the alternative hypothesis 
of the multiverse counts as an undercutting defeater ${ }^{12}$ for the alleged evidential connection between 'fine-tuning' and 'fine-tuner' (or 'designer').

In the next section, then, I articulate an objection to the FTA that targets the alleged evidential connection between 'fine-tuner' (or 'designer') and 'a supernatural designer possessing power and knowledge sufficient to create a universe with life and a strong motive for doing so'. ${ }^{13}$ If this alleged evidential connection is undercut, then the FTA is a weak argument for the existence of God.

\section{A new objection against the FTA}

Unlike Objection 1, which targets the alleged evidential connection between 'fine-tuning' and 'fine-tuner' (or 'designer'), my objection targets the alleged evidential connection between 'finetuner' (or 'designer') and 'supernatural designer of immense power and knowledge', as the design hypothesis would have it. The objection appeals to the simulation hypothesis. According to the simulation hypothesis, we humans currently live in a computer simulation. ${ }^{14}$ If the simulation hypothesis is true, then it provides a straightforward explanation for fine-tuning. The universe is fine-tuned for life because it was programmed that way. The universe was programmed for life, however, not by 'a supernatural designer of immense power and knowledge' but by human beings like us. Although these programmers are humans that have reached a 'posthuman' stage of civilization, and thus are technologically more advanced than we are, they are not immensely powerful and knowledgeable in the way that the supernatural designer of the design hypothesis is supposed to be. 
If this is correct, then in addition to the 'chief rival explanation for fine-tuning', namely, the multiverse hypothesis, ${ }^{15}$ another rival explanation is the simulation hypothesis. It is worth noting, however, that the simulation hypothesis and the multiverse hypothesis are not incompatible. It may be the case that the multiverse hypothesis, if true, explains why there is a universe at all, given that there are many (perhaps infinitely many) universes. But that is consistent with the simulation hypothesis, which, if true, would explain why this (our) universe is a simulation. ${ }^{16}$

Accordingly, just as the multiverse hypothesis is an undercutting defeater for the alleged evidential connection between 'fine-tuning' and 'fine-tuner' (or 'designer'), the simulation hypothesis is an undercutting defeater for the alleged evidential connection between 'fine-tuner' (or 'designer') and 'supernatural designer of immense power and knowledge'. If the simulation hypothesis is true, then the 'designers' are neither divine nor supernatural.

Of course, if the simulation hypothesis is true, then we humans are not 'alive' in the ordinary sense of the term. But that is beside the point. For what we would observe if the simulation hypothesis were true is exactly what we observe now. That is, if the simulation hypothesis is true, it would still appear to us that the universe is fine-tuned for life, i.e., it would appear to us that $\mathrm{E}$ is the case, and it would still appear to us that many of the initial conditions and free parameters of a universe need to be finely tuned in order for the development of life in that universe to be possible, i.e., it would appear to us that $\mathrm{K}$ is the case. In that respect, the simulation hypothesis, if true, would explain the phenomena just as well as the design hypothesis would, if it were true. 
Some might object that the simulation hypothesis is implausible, Bostrom's arguments in support of the simulation hypothesis notwithstanding. For present purposes, however, the question is whether the simulation hypothesis is less plausible than the design hypothesis. To answer this question, these two competing explanations for fine-tuning need to be judged relative to generally accepted criteria of selection. Philosophers of science have offered the following key criteria for selecting between competing explanations ${ }^{17}$ :

Unification: As a general rule of thumb, choose the explanation that explains the most and leaves the least unexplained things.

Coherence: As a general rule of thumb, choose the explanation that is consistent with background knowledge.

Simplicity: As a general rule of thumb, choose the least complicated explanation, i.e., the one that posits the least causal sequences and entities, and that goes beyond the evidence the least.

Testability: As a general rule of thumb, choose the explanation that yields independently testable predictions.

Now, in terms of unification, the design hypothesis and the simulation hypothesis seem to account for the phenomena of fine-tuning equally well. However, the design hypothesis raises many unanswered questions that the simulation hypothesis does not. Some of these unanswered questions are the following: Where did the supernatural designer come from? Why is there one 
supernatural designer rather than a few (or many)? Why did the supernatural designer want to have life in the universe at all? Moreover, the simulation hypothesis arguably explains phenomena other than fine-tuning, such as quantum indeterminacy. ${ }^{18}$ To that extent, its unification power exceeds that of the design hypothesis.

In terms of coherence, the simulation hypothesis seems more consistent with our background knowledge than the design hypothesis is. As far as supernatural entities are concerned, our background knowledge is quite limited compared to what we know about ourselves and our technology, particularly our computational resources. ${ }^{19}$

In terms of simplicity, the design hypothesis postulates the existence of a being that is different in kind from anything else in the universe, whereas the simulation hypothesis says that the designers are not different from us in kind, only in degree (specifically, degree of technological development). So the design hypothesis postulates the existence of a kind of entity that is not quite consistent with our background knowledge. In general, if 'entities should not be multiplied beyond necessity', then the simulation hypothesis is the simpler one.

Finally, and more importantly, in terms of testability, there does not seem to be an independent way to test the design hypothesis. The design hypothesis predicts fine-tuning, of course, but so does the simulation hypothesis. To choose between the two, then, we need a way to test these hypotheses independently of the explanandum (i.e., fine-tuning). One might think that there is no way to test the design hypothesis, even in principle, given that it postulates the existence of a supernatural entity, and thus it goes beyond the scope of scientific inquiry, which deals with the natural, not the supernatural. Be that as it may, there does not seem to be a way to test the design hypothesis independently the explanandum (i.e., fine-tuning). 
On the other hand, the simulation hypothesis makes at least one prediction that can be tested independently of the explanandum. If the simulation hypothesis is true, then there would be similarities between our universe and computer simulations like The Sims. One such similarity that can be directly tested is whether the universe is made of 'pixels', just like a computer simulation. Any computer-generated image can be broken down into small parts or pixels. Similarly, any chunk of matter can be broken down into small parts or atoms (and further into subatomic particles). To put it somewhat more technically, if the simulation hypothesis is true, then there should be a cut off in the spectrum of high-energy particles. In fact, there may be such a cut off in the energy of cosmic ray particles, which is known as the Greisen-Zatsepin-Kuzmin or GZK cut off. ${ }^{20}$

For these reasons, the simulation hypothesis not only undercuts the alleged evidential connection between 'fine-tuner' (or 'designer') and 'supernatural designer of immense power and knowledge' but it also outperforms the design hypothesis when the two are evaluated in terms of theoretical virtues, such as unification, coherence, simplicity, and testability. For present purposes, I will not argue any further for the superiority of the simulation hypothesis over the design hypothesis but rather rest content with showing (as I think I have) that the simulation hypothesis, which provides an alternative explanation for fine-tuning, undercuts the alleged evidential connection between 'designer' and 'supernatural designer of immense power and knowledge' in much the same way that the multiverse hypothesis undercuts the alleged evidential connection between 'fine-tuning' and 'fine-tuner' (or 'designer'). If this is correct, then the FTA is a weak argument for the existence of 'a supernatural designer possessing power and knowledge sufficient to create a universe with life and a strong motive for doing so' ${ }^{21}$ 


\section{Conclusion}

In this paper, I have proposed the simulation hypothesis as another explanation, in addition to the multiverse hypothesis and the design hypothesis, for fine-tuning. I have argued that the simulation hypothesis undercuts the alleged evidential connection between 'designer' and 'supernatural designer of immense power and knowledge' in much the same way that the multiverse hypothesis undercuts the alleged evidential connection between 'fine-tuning' and 'fine-tuner' (or 'designer'). If this is correct, then the FTA is a weak argument for the existence of God. Insisting that the simulation hypothesis is implausible will not do because when the simulation hypothesis and the design hypothesis are evaluated in terms of generally accepted criteria of selection, the former emerges as the better (i.e., simpler and more coherent) explanation for fine-tuning. Moreover, the unification and predictive powers of the simulation hypothesis exceed that of the design hypothesis.

\section{Notes}

\footnotetext{
${ }^{1}$ N. A. Manson, 'The fine-tuning argument', Philosophy Compass, vol. 4 (2009), pp. 271-286.

${ }^{2}$ Manson, 'The fine-tuning argument', p. 272.

${ }^{3}$ Manson, 'The fine-tuning argument', p. 273.

${ }^{4}$ L. Smolin, The Life of the Cosmos (New York: Oxford University Press, 1997).

5 D. Bradley, 'Multiple universes and observation selection effects', American Philosophical Quarterly, vol. 46 (2009), pp. 61-72.

${ }^{6}$ P. Davies, P. 1992. The Mind of God: The Scientific Basis for a Rational World (New York: Simon \& Schuster, 1992), p. 204.

${ }^{7}$ R. White, 'Fine-tuning and multiple universes', Noûs, vol. 34 (2000), pp. 260-276.

${ }^{8}$ N. A. Manson and M. J. Thrush, 'Fine-tuning, multiple universes, and the "this universe" objection', Pacific Philosophical Quarterly, vol. 84 (2003), pp. 67-83.

${ }^{9}$ J. Weisberg, 'A note on design: what's fine-tuning got to do with it?' Analysis, vol. 70 (2010), pp. 431-438.

${ }^{10}$ R. White, 'What fine-tuning's got to do with it: a reply to Weisberg', Analysis, vol. 71 (2011), pp. 676-679.
} 
${ }^{11}$ J. Weisberg, 'The argument from divine indifference', Analysis, vol. 72 (2012), pp. 707-714. D. Bradley, 'Weisberg on design: what fine-tuning's got to do with it', Erkenntnis, vol. 77 (2012), pp. 435-438.

${ }^{12}$ J. L. Pollock and J. Cruz, Contemporary Theories of Knowledge (Lanham, MA: Rowman \& Littlefield, 1999), p. 196.

${ }^{13}$ Manson and Thrush, 'Fine-tuning, multiple universes, and the "this universe" objection', p. 68.

${ }^{14}$ N. Bostrom, 'Are you living in a computer simulation?' The Philosophical Quarterly, vol. 53 (2003), pp. 243-255. N. Bostrom, 'A patch for the simulation argument', Analysis, vol. 71 (2011), pp. 54-61.

${ }^{15}$ R. Holder, 'Fine-tuning and the multiverse,' Think, vol. 4 (2006), pp. 49-60.

${ }^{16}$ Thanks to Marcus Arvan for this point.

${ }^{17}$ M. Mizrahi, 'Why the ultimate argument for scientific realism ultimately fails', Studies in History and Philosophy of Science, vol. 43 (2012), pp. 132-138.

${ }^{18}$ M. Arvan, 'A new theory of free will', The Philosophical Forum, vol. 44 (2013), pp. 1-48.

${ }^{19}$ N. Bostrom, 'The simulation argument: some explanations', Analysis, vol. 69 (2009), pp. 458-461.

${ }^{20}$ S. R. Beane, Z. Davoudi, and M. J. Savage, 'Constraints on the universe as a numerical simulation', arXiv:1210.1847v2 [hep-ph] (2012). See also Arvan, 'A new theory of free will', pp. 39-42.

${ }^{21}$ Manson and Thrush, 'Fine-tuning, multiple universes, and the "this universe" objection', p. 68. 